\title{
What do the lineage tracing studies tell us? Consideration for hematopoietic stem cell origin, dynamics, and leukemia-initiating cells
}

\author{
Nathalia Azevedo Portilho ${ }^{1} \cdot$ Michihiro Kobayashi $^{1} \cdot$ Momoko Yoshimoto $^{1}$ (I) \\ Received: 31 August 2018 / Accepted: 18 September 2018 / Published online: 27 September 2018 \\ (c) The Japanese Society of Hematology 2018
}

\begin{abstract}
The recent advance of technologies enables us to trace the cell fate in vivo by marking the cells that express the gene of interest or by barcoding them at a single cell level. Various tamoxifen-inducible Cre-recombinase mice combined with Rosa-floxed lines are utilized. In this review, with the results revealed by lineage tracing assays, we re-visit the long-standing debate for the origin of hematopoietic stem cells in the mouse embryo, and introduce the view of native hematopoiesis, and possible leukemic-initiating cells emerged during fetal stages.
\end{abstract}

Keywords Hematopoietic stem cells $\cdot$ Mouse embryo $\cdot$ Yolk sac $\cdot$ AGM $\cdot$ Lineage tracing

\section{Introduction}

The hematopoietic stem cells (HSCs) in the bone marrow $(\mathrm{BM})$ are providing all types of blood cells throughout life by their self-renewal and multilineage differentiation abilities. Searching for the temporal and spatial origin of HSCs during ontogeny has long been a focus of research in the field of developmental hematology. Because HSCs are defined only by transplantation assay as long-term (more than 6 months) multilineage repopulating cells, embryonic tissues at various stage of embryos were transplanted into lethally irradiated adult mice. The first HSCs detectable by transplantation assays are found at embryonic day (E) 10-11 in the aorta-gonad-mesonephros (AGM) region of the mouse embryo [1, 2]. However, prior to E10, many hematopoietic activities are first observed in the extra-embryonic yolk sac (YS) from E7.5, such as primitive hematopoiesis including the production of primitive erythrocytes, megakaryocytes, and macrophages [3]. In the adult HSCs, all blood

Momoko Yoshimoto

Momoko.Yoshimoto@uth.tmc.edu

1 Center for Stem Cell and Regenerative Medicine at Institute of Molecular Medicine, McGovern Medical School, University of Texas Health Science Center at Houston, 1825 Pressler Street, Houston 77030, USA cells are considered to be products of HSCs; therefore, it was assumed that there should be a stem cell potential prior to E10, which was not detectable by direct transplantation assays. To examine the HSC potential, various organ cultures and co-cultures with stromal cells have been performed. The lymphoid potential was measured as one of HSC potential, with both YS and paraaortic splanchnopleural (P-Sp) region [4-9]. Cumano et al. reported a lymphoid repopulating ability of P-Sp cells at a pre-circulation stage after organ culture [4], while Matsuoka et al. showed multilineage repopulating ability of E8.5 YS after co-culture with AGM-derived stromal cells [10]. More recently, Medvinsky's group identified precursors of HSCs that express VE-cadherin and c-kit in the AGM region at E10.5, and that obtain HSC activity only after aggregation culture with OP9 [11, 12]. Same results were confirmed by different groups using Delta-like 1 expressing OP9 [13] or Akt-expressing AGM-derived endothelial cell lines [14]. Weissman reported that YS has HSC activity once it was transplanted into fetus intrauterinally [15]. Yoder et al. used neonatal mice as recipients and showed E9 YS and P-Sp cells repopulated neonatal mice [16]. All these results came from different co-culture systems and different recipient environments [17] (adult vs. neonates/embryos) thus showing a limitation of assays.

To avoid in vitro culture and transplantation assays to evaluate fundamental hematopoietic characteristics, lineage 
tracing studies have been developed. Cre-recombinant gene is inserted after a promoter region of the gene of interest, and by crossing them with mice carrying a fluorescent protein floxed allele, cells expressing the gene turn into positive for the fluorescent, thus one can trace the progeny of the cells that have expressed the gene of interest before (Fig. 1). Even labeling of cells at a specific time point is enabled by combining with estrogen receptor-induced Cre-system. By injecting tamoxifen at one time point, the cells expressing a gene of interest is labeled at that time. Utilizing this lineage tracing system, YS potential to produce adult HSCs has been demonstrated, revisiting the long-lasting debate of the origin of HSCs in the mouse embryo.

In this review, we introduce key literature that demonstrates the HSC potential in the YS and AGM region by lineage tracing system, and also introduce some models to examine HSC multipotency and clonality in adult mice. Finally, we will consider the possible fetal origin of leukemia utilizing lineage tracing models.

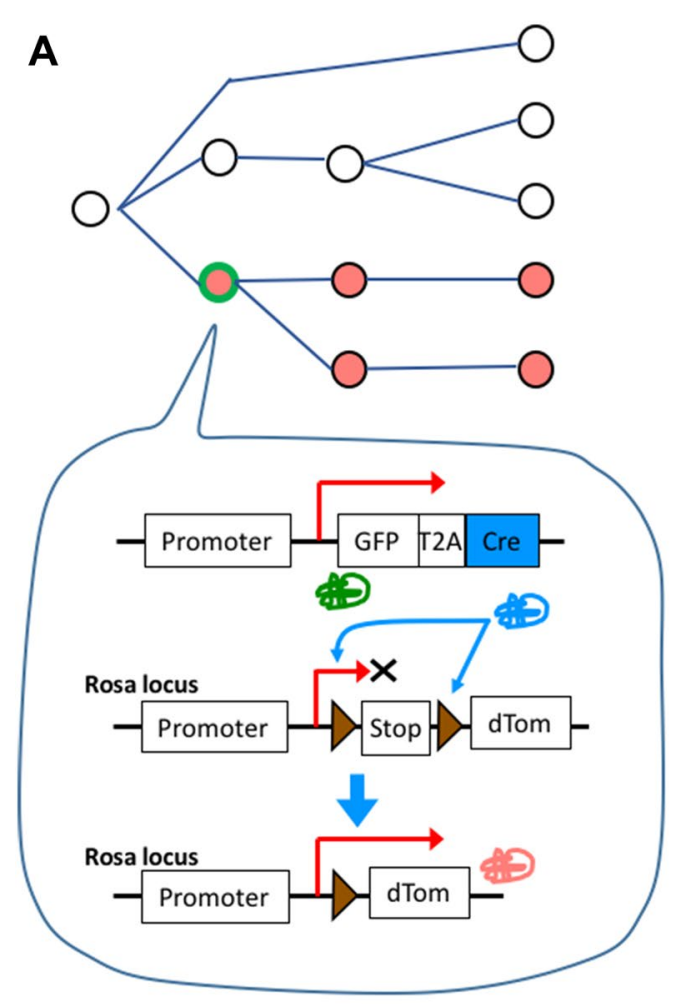

Fig. 1 a Schematic representation of the genetic elements in the standard Cre-loxP system. Cre-recombinase is constitutively expressed under the control of a specific gene promoter together with GFP. In those cells, Cre recombine loxP sites and remove Stop cassette in the ubiquitously expressed reporter construct. Then, dTom (or YFP or Lacz) become positive in these cells and all their progenies. b Cre is fused to a tamoxifen-inducible mutated estrogen recep-

\section{Endothelial origin of hematopoietic cells in the embryo}

The concept of the endothelial origin of hematopoietic cells during ontogeny has been proposed based on the observation that blood cells looked like "budding" from the endothelial wall of the dorsal aorta [18]. Runx1 is a critical transcriptional factor for definitive hematopoiesis, and its knockout embryos display lack of definitive hematopoiesis including the hematopoietic stem cells and diminishment of blood cell "budding" in the dorsal aorta. This observation led a hypothesis that the blood cell budding seen in the dorsal aorta (which expresses runx1) contains HSCs and represents an endothelial-hematopoietic transition (EHT). The concept of blood-forming endothelial cells (named hemogenic endothelial cells: HECs) were also proved by in vitro co-culture with stromal cells where B- and T-cells were produced by sorted endothelial cells from YS and/or P-Sp [8, 9, 18]. To prove this concept in vivo, endothelial-lineage tracing mouse has been developed. The VE-cadherin (VE-cad) is expressed

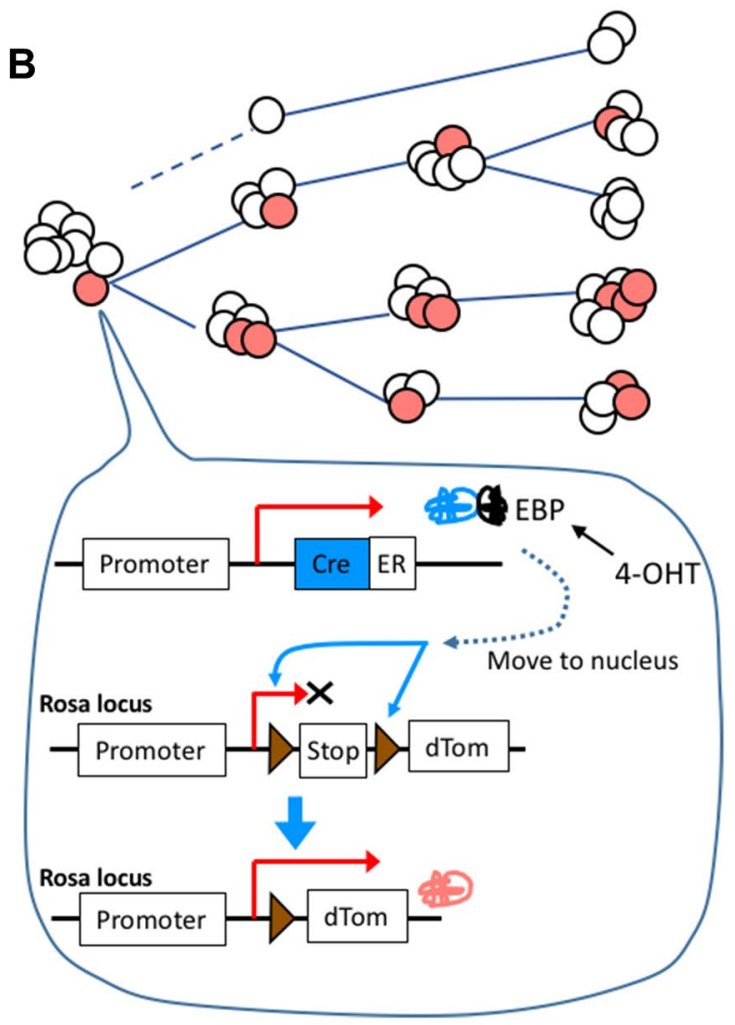

tor (CreERT2). CreERT2 is expressed under the control of a specific gene promoter. The cells expressing the gene keep CreERT2 inactive in the cytoplasm. Upon binding of tamoxifen or its active metabolite 4-hydroxy-tamoxifen(4-OHT), CreERT2 is released and translocates to the nucleus. Then, Cre recombines the loxP sites and remove the Stop cassette enabling dTom expression 
almost exclusively in the conceptus vasculature except for the heart and a brief detection in the mesoderm of yolk sac at E7.5 [19]. Zovein et al. crossed VE-cad Cre-transgenic mice with Rosa26R (R26)-Lacz reporter line so that VE-cad progeny became Lacz positive and demonstrated the endothelial origin of definitive HSCs that persist into adult life [20]. Using tamoxifen-inducible VE-cad Cre mice which label VE-cad+ expressing cells as YFP+ permanently at a time of tamoxifen injection, they also proved the point that the intra-aortic clusters ("budding cells" from endothelial cells) marked at E10.5-11.5 are the ancestor of all blood lineages including HSCs in the adult BM.

\section{AGM-derived HSCs}

Following the report of VE-cad lineage tracing study above, Chen et al. further asked a question about a role of Runx 1 expressed in the HEC [21]. First, they generated VE-cad Cre: R26R-YFP line to trace VE-cad+ cells and confirmed that VE-cad+ cells produced all blood cells in the E15 fetal liver and adult BM. Next, they deleted the Runx1 gene in VE-cad+ cells by generating VE-cad Cre: $\operatorname{runx} 1^{\text {flox/flox }}$ mice. Runx1 deletion specifically in the VE-cad+ endothelial cells induced diminishment of colony-forming cells, c-kit positive cells, intra-aortic clusters, and HSCs. Importantly, hematopoietic specific deletion (Vav-Cre) of runx 1 did not affect the emergence of colony-forming cells and HSCs. Thus, utilizing the lineage tracing model and lineage-specific runx 1 deletion, Chen et al. showed HSCs are derived from VEcad+ endothelial cells, and runx 1 is essential for endothelial-hematopoietic transition, but not thereafter.

However, one caveat is that these reports do not define the role of HECs specifically in the AGM region, but instead define all HECs in YS, placenta, and vitelline arteries in addition to AGM. In this sense, there are no lineage tracing reports that prove the AGM region as a specific HSC origin. Time-lapse imaging utilizing the Ly6A (Sca-1) reporter mice revealed the emergence of GFP+ Sca-1+ cells (assuming HSCs) from the endothelial cells in the dorsal aorta [22], but this did not necessarily mean the emergence of functional HSCs.

\section{YS-derived HSCs}

YS is the first site to produce hematopoietic cells in the embryo, thus had traditionally been considered to be a place to produce HSCs until the detection of the first adult repopulating HSCs in the AGM region at E10.5. Since then, even though YS possesses abundant colony-forming units (erythro-myeloid progenitors) and $\mathrm{T}$ and $\mathrm{B}$ lymphoid potentials, YS often shows less engraftment ability in recipient mice; therefore, YS is disregarded as a site of HSC production in many recent pieces of literature. After a long-standing debate about the origin of HSC, YS vs. AGM region, Nishikawa and Samokhvalov group developed a fascinating mouse model to detect the origin of HSCs in the embryo by lineage tracing and demonstrated that YS cells at E7.5 contributed to adult HSCs [23].

Samokhvalov et al. produced a knock-in mouse containing the tamoxifen-inducible MER-Cre-MER gene inserted under the control of the Runx 1 locus. The authors crossed heterozygous runx $1^{\text {Mer-Cre-Mer }}$ mice with Rosa26-lacZ or Rosa26-eYFP lines. This system enables them to trace the progeny of runx 1 expressing cells at a specific time point temporally and spatially (Fig. 1b). First, the authors confirmed that runx1 positive cells at E7.5-8.25 were restricted to YS; thus the progeny of E7.5 runx1 positive cells were considered to be YS-derived. By tamoxifen injection at E7.5, YFP+ cells were detected among the cKit+ Ter119+ definitive erythroid progenitors, myeloid cells, CD34+ c-kit+ HSC/HPCs in the fetal liver at E12.5, as well as erythro-myeloid and lymphoid lineages in the fetal liver and thymus at E16.5. Post-natal analysis revealed the persistence of YS-derived cells in the adult peripheral blood and the $\mathrm{BM}$, including in the CD34KSL HSC population, up to 12 months. In addition, they showed E7.5 labeled $\beta$-gal+ intra-aortic clusters at E10.5 and umbilical arteries and veins at E11.5, suggesting that YS runx 1+ cells at E7.5 may have migrated to the AGM and other vessel regions and contributed to a part of HSCs.

Subsequently, with the notion above that HSCs produced in the AGM region may ultimately be derived from E7.5 YS runx1+ cells, Samokhvalov's group developed "rescue" tracing system that determines which day of runx1 expression is indispensable for the HSC production in vivo [24]. In their system, Tamoxifen injections at E6.5 and E7.5 efficiently rescued runx1-null phenotype in embryos or newborns (80-100\%) while recombination at later stages (E8.0, E8.5, E9.5, or E10.5) failed to prevent severe hemorrhage and fetal liver anemia in embryos. Also, E16.5 fetal liver HSCs rescued at E7.5 were able to long-term engraftment after transplantation. Thus, although runx 1 is known to be critical for HSC emergence at E10.5, runx1 expression at E7.5 is indispensable than at E10.5 for the HSC generation. Taken together, the authors proposed that runx 1 expressing cells in extra-embryonic mesoderm at E7.5 are the ontogenic source of HSC precursors that become adult HSCs in a later stage of embryos.

These papers by the Nishikawa and Samokhvalov group were the first to reveal the extra-embryonic cell contribution to long-term adult hematopoiesis, previously hidden in transplantation assays, by non-invasive lineage tracing analysis. 
One of the confounding factors that prevents the research for the HSC origin in the mouse embryo is a circulation that may mix cells from different origins that share many surface markers and gene expressions. Therefore, genes and markers that are specifically expressed in either YS or P-Sp/AGM have been sought for, and Lyve-1 is exclusively expressed in the YS endothelium until E10.5 while only minimum expression was detected in placenta and embryo vessels [25, 26]. Utilizing Lyve-1 Cre model, Mikkola's group demonstrated that up to $40 \%$ of HSCs in the fetal liver and adult BM were YS-derived [27]. In addition, analysis of heartbeat-deficient Ncx 1-/- embryos confirmed that YS was the primary source of Lyve1+ cells. One caveat is that the degree of the contribution of minimum Lyve-1+ cells in the AGM region and placenta to adult hematopoiesis is still unknown.

Taken together, it seems that Runx 1+ cells at E7.5 extraembryonic mesoderm are essential precursors of HSCs, but it is still unknown how much AGM-derived ECs contribute to HSC in the adult BM, while YS is estimated to contribute up to 40-50\% [27], although AGM cells at E10-11 engraft in the recipient mice upon transplantation. It would be reasonable to consider that all ECs in YS, AGM, vitelline arteries and placenta produce HSC precursors and HSCs at various time points in vivo.

\section{Studies on HSC-derived hematopoiesis by barcoding and lineage tracing in adult mice}

Once HSCs seed the BM around birth, HSC-derived hierarchical hematopoiesis establishes. In the post-natal BM, LT-HSC has been purified at a single cell level, as lin-Sca1+c-kit+CD135-CD34-CD150+CD48-EPCR+ proved by transplantation assays [28, 29]. However, the conditioning before transplantation such as irradiation destroys the BM environment and normal hematopoiesis in steady state. To understand the dynamics of native hematopoiesis, Camargo's group developed a cell-marking system at single cell level by a doxycycline-inducible transposon (Tn)-random integration [30]. In this system, after doxycycline injection, the Tn integrates elsewhere with Ds-Red reporter-on, so that Ds-Red positive cells can be identified their clonality by PCR and sequencing of their Tn integration sites. With this powerful system, the authors revealed the unexpected smaller contribution of LT-HSCs to steady-state hematopoiesis in vivo, such as only $5 \%$ of LT-HSC clones shared with mature blood cells and only less than $5 \%$ of LT-HSC clones shared with MPPs. They have recently updated this system and demonstrated that megakaryocyte clones were not shared with other lineages in the $\mathrm{PB}$, but were shared exclusively with LT-HSCs in the BM. This indicates that megakaryocytes are an immediate progeny of LT-HSCs and compatible with reports that megakaryocyte-biased HSCs [31].

Another fascinating report has come out developing polylox barcoding system [32]. Rodewald's group generated polylox cassettes composed of ten loxP sites that yield several hundred thousand barcodes by random incomplete recombination and inversions. Combining with Tie $2^{\mathrm{Mer}-\mathrm{Cre}-\mathrm{Mer}}$ mice, they successfully induced polylox barcoding in HSC precursors at the embryonic stage and demonstrated that adult BM HSCs contain many clones derived from embryonic precursors. The clonal correlation between erythrocyte and myeloid lineages, and $\mathrm{T}$ and $\mathrm{B}$ lymphocytes supported the concept of the traditional hematopoietic tree that segregates erythro-myeloid progenitors from common lymphoid progenitors. Importantly, B-1a cells that are considered to be fetal-derived and that could before HSC-independent lineage are clearly separated from other lymphocyte subsets.

Another strategy of HSC lineage tracing is specifically labeling LT-HSC. Based on the results of single cell RNA sequencings of LT-HSCs, genes such as Fgd5 [33], Pdzk1ip1 [34], Krt18 [35], and Krt7 [36] have been utilized. Sawai et al. showed Pdzk1ip1 was expressed exclusively in BM LTHSC, and Pdzk1ipCreER mice marked LT-HSCs after three sequential day tamoxifen injections [34]. Tomato + mature blood cells appeared at $60 \%$ of each lineage by 36 weeks, and as expected, fetal-derived immune cells such as B-1a cells and brain macrophages were not labeled very well $(<5 \%)$. Thus, HSC-specific lineage tracing system enabled to show the differentiation kinetics of HSC progeny and supported the presence of HSC-independent immune cells.

\section{Prenatal leukemia-initiating cells using lineage tracing study}

The recent development of whole genome sequencing revolutionized to depict the multi-step progressions of leukemia in an individual patient, where just simple sequential peripheral blood sampling gives us detailed information from clonal hematopoiesis to aggressive populations having several clonal evolutions [37, 38]. Also clarifying leukemia stem cell (LSC)/initiating cell (LIC) has been widely investigated using a wide variety of leukemia model or patient samples, which has turned out that immature progenitor cells (MPP or c-Kit+ cells) are shown to be LIC in the adult cases by utilizing mainly Mx1-cre systems [39]. In view of intrauterine of lineage tracing for hematopoiesis, prenatal LIC will be discussed here, in which a precise mouse model to initiate neoplastic hematopoiesis at a specific stage of the embryo will be required. About a decade ago, sequential development of clonal leukemic evolution from LIC was already reported by tracking unique leukemia translocation from patient's bone marrow to the prenatal stage (cord blood) 
[40, 41]. This type of leukemia harboring ETV6-Runx1 is common in childhood B-precursor leukemia and less aggressive, allowing us to observe a pre-leukemic phase. Indeed, ETV6-Runx1 positive B-progenitors (LICs) were present in cord blood, known to be required for leukemia initiation, but insufficient for progressing to overt leukemia by itself [42]. This is a clear evidence of the LIC in the prenatal hematopoiesis, but it is still unknown whether the LICs found at a prenatal period are derived from HSCs or HSC-independent lymphoid progenitors. Similarly, transient abnormal myelopoiesis (TAM) is a common perinatal condition in Down's syndrome and known to be proceeding to myeloid leukemia in a part of TAM patients. As myeloid progenitors with GATA1 mutation is identified as LIC [43, 44], the origin of TAM initiating cells has been unknown. Recently, Tarnawski et al. reported a well-depicted mouse model resembling clinical phenotype of juvenile myelomonocytic leukemia (JMML) [45], a unique disease that shows clonal myeloid proliferation with involvement of cytokine receptor/Ras signaling in the young child. They created two mouse models in regards to major responsible genes, Ras and Ptpn11, respectively. In the first model, Kras mutant clone is initiated at Flt 3 positive MPP in the fetal liver and the mutant mice display a remarkable abdominal distention within 3 weeks postnatally due to hepato-splenomegaly, which resembles clinical phenotype of JMML [45]. On the other hand, with a combination of ras and major hematopoietic cre-driver, Vavcre/Mx1-cre, it has been difficult to recapitulate the similar phenotype to human patients due to embryonic lethality $[46,47]$. Although another model was also reported by the same group with Flt3-cre and mutant Ptpn11, its phenotype was very indolent compared to ras-based model [48]. Those results suggest that Flt $3^{+}$MPP in the FL is the LIC of JMML and the combination of appropriate strength of oncogenic driver in the proper cell stage will be required for disease initiation.

Overall, since the development of lineage tracing study in a targeted spatiotemporal manner, the more detailed characterization of LIC will be achieved to visualize the consequence of LIC to clonal evolution in the non-transplant native mouse model with prospective fashion.

\section{Conclusion}

In this paper, lineage tracing studies in the studies of the origin of HSCs, native HSC-derived hematopoiesis in the adult, and the LIC have been discussed. Combination of lineage tracing assays and clonal assays (such as barcoding) in vivo will enable us to understand the kinetics of various hematopoietic lineages emerging during the ontogeny.

Acknowledgements This study is supported by NIAID R01AI121197.

\section{References}

1. Muller AM, Medvinsky A, Strouboulis J, Grosveld F, Dzierzak E. Development of hematopoietic stem cell activity in the mouse embryo. Immunity. 1994;1:291-301.

2. Medvinsky A, Dzierzak E. Definitive hematopoiesis is autonomously initiated by the AGM region. Cell. 1996;86:897-906.

3. Palis J, Robertson S, Kennedy M, Wall C, Keller G. Development of erythroid and myeloid progenitors in the yolk sac and embryo proper of the mouse. Development. 1999;126:5073-84.

4. Cumano A, Dieterlen-Lievre F, Godin I. Lymphoid potential, probed before circulation in mouse, is restricted to caudal intraembryonic splanchnopleura. Cell. 1996;86:907-16.

5. Godin I, Dieterlen-Lievre F, Cumano A. Emergence of multipotent hemopoietic cells in the yolk sac and paraaortic splanchnopleura in mouse embryos, beginning at 8.5 days postcoitus. Proc Natl Acad Sci USA. 1995;92:773-7.

6. Yokota T, Huang J, Tavian M, Nagai Y, Hirose J, ZunigaPflucker JC, et al. Tracing the first waves of lymphopoiesis in mice. Development. 2006;133:2041-51.

7. Nishikawa SI, Nishikawa S, Kawamoto H, Yoshida H, Kizumoto M, Kataoka H, et al. In vitro generation of lymphohematopoietic cells from endothelial cells purified from murine embryos. Immunity. 1998;8:761-9.

8. Yoshimoto M, Montecino-Rodriguez E, Ferkowicz MJ, Porayette P, Shelley WC, Conway SJ, et al. Embryonic day 9 yolk sac and intra-embryonic hemogenic endothelium independently generate a B-1 and marginal zone progenitor lacking B-2 potential. Proc Natl Acad Sci USA. 2011;108:1468-73.

9. Yoshimoto M, Porayette P, Glosson NL, Conway SJ, Carlesso $\mathrm{N}$, Cardoso AA, et al. Autonomous murine T-cell progenitor production in the extra-embryonic yolk sac before HSC emergence. Blood. 2012;119:5706-14.

10. Matsuoka S, Tsuji K, Hisakawa H, Xu MJ, Ebihara Y, Ishii T, et al. Generation of definitive hematopoietic stem cells from murine early yolk sac and paraaortic splanchnopleures by aorta-gonad-mesonephros region-derived stromal cells. Blood. 2001;98:6-12.

11. Rybtsov S, Sobiesiak M, Taoudi S, Souilhol C, Senserrich J, Liakhovitskaia A, et al. Hierarchical organization and early hematopoietic specification of the developing HSC lineage in the AGM region. J Exp Med. 2011;208:1305-15.

12. Taoudi S, Gonneau C, Moore K, Sheridan JM, Blackburn CC, Taylor E, et al. Extensive hematopoietic stem cell generation in the AGM region via maturation of VE-cadherin+ CD45+ predefinitive HSCs. Cell Stem Cell. 2008;3:99-108.

13. Zhou F, Li X, Wang W, Zhu P, Zhou J, He W, et al. Tracing haematopoietic stem cell formation at single-cell resolution. Nature. 2016;533:487-92.

14. Hadland BK, Varnum-Finney B, Mandal PK, Rossi DJ, Poulos MG, Butler JM, et al. A common origin for B-1a and B-2 lymphocytes in clonal pre-hematopoietic stem cells. Stem Cell Rep. 2017;8:1563-72.

15. Weissman IL, Papaioannou V, Gardner R. Fetal hematopoietic origin of the adult hematolymphoid system. In: Clarkson B, Mark P, Till J, editors. Differentiation of normal and neoplastic hematopoietic cells. Cold Spring Harbor conferences on cell proliferation. New York: Cold Spring Harbor Laboratory; 1978. pp. 33-47.

16. Yoder MC, Hiatt K, Dutt P, Mukherjee P, Bodine DM, Orlic D. Characterization of definitive lymphohematopoietic stem cells in the day 9 murine yolk sac. Immunity. 1997;7:335-44.

17. Arora N, Wenzel PL, McKinney-Freeman SL, Ross SJ, Kim PG, Chou SS, et al. Effect of developmental stage of HSC and recipient on transplant outcomes. Dev Cell. 2014;29:621-8. 
18. North T, Gu TL, Stacy T, Wang Q, Howard L, Binder M, et al. $\mathrm{Cbfa} 2$ is required for the formation of intra-aortic hematopoietic clusters. Development. 1999;126:2563-75.

19. Breier G, Breviario F, Caveda L, Berthier R, Schnürch H, Gotsch $\mathrm{U}$, et al. Molecular cloning and expression of murine vascular endothelial-cadherin in early stage development of cardiovascular system. Blood. 1996;87:630-41.

20. Zovein AC, Hofmann JJ, Lynch M, French WJ, Turlo KA, Yang Y, et al. Fate tracing reveals the endothelial origin of hematopoietic stem cells. Cell Stem Cell. 2008;3:625-36.

21. Chen MJ, Yokomizo T, Zeigler BM, Dzierzak E, Speck NA. Runx 1 is required for the endothelial to haematopoietic cell transition but not thereafter. Nature. 2009;457:887-91.

22. Boisset JC, van Cappellen W, Andrieu-Soler C, Galjart N, Dzierzak E, Robin C. In vivo imaging of haematopoietic cells emerging from the mouse aortic endothelium. Nature. 2010;464:116-20.

23. Samokhvalov IM, Samokhvalova NI, Nishikawa SI. Cell tracing shows the contribution of the yolk sac to adult haematopoiesis. Nature. 2007;446:1056-61.

24. Tanaka Y, Hayashi M, Kubota Y, Nagai H, Sheng G, Nishikawa S-I, et al. Early ontogenic origin of the hematopoietic stem cell lineage. Proc Natl Acad Sci. 2012;109:4515-20.

25. Van Handel B, Montel-Hagen A, Sasidharan R, Nakano H, Ferrari $\mathrm{R}$, Boogerd $\mathrm{CJ}$, et al. $\mathrm{Scl}$ represses cardiomyogenesis in prospective hemogenic endothelium and endocardium. Cell. 2012;150:590-605.

26. Gordon EJ, Gale NW, Harvey NL. Expression of the hyaluronan receptor LYVE-1 is not restricted to the lymphatic vasculature; LYVE-1 is also expressed on embryonic blood vessels. Dev Dyn. 2008;237:1901-9.

27. Lee LK, Ghorbanian Y, Wang W, Wang Y, Kim YJ, Weissman IL, et al. LYVE1 marks the divergence of yolk sac definitive hemogenic endothelium from the primitive erythroid lineage. Cell Rep. 2016;17:2286-98.

28. Yamamoto R, Morita Y, Ooehara J, Hamanaka S, Onodera M, Rudolph KL, et al. Clonal analysis unveils self-renewing lineagerestricted progenitors generated directly from hematopoietic stem cells. Cell. 2013;154:1112-26.

29. Benz C, Copley MR, Kent DG, Wohrer S, Cortes A, Aghaeepour $\mathrm{N}$, et al. Hematopoietic stem cell subtypes expand differentially during development and display distinct lymphopoietic programs. Cell Stem Cell. 2012;10:273-83.

30. Sun J, Ramos A, Chapman B, Johnnidis JB, Le L, Ho YJ, et al. Clonal dynamics of native haematopoiesis. Nature. 2014;514:322-7.

31. Carrelha J, Meng Y, Kettyle LM, Luis TC, Norfo R, Alcolea V, et al. Hierarchically related lineage-restricted fates of multipotent haematopoietic stem cells. Nature. 2018;554:106-11.

32. Pei W, Feyerabend TB, Rössler J, Wang X, Postrach D, Busch K, et al. Polylox barcoding reveals haematopoietic stem cell fates realized in vivo. Nature. 2017;548:456-60.

33. Gazit R, Mandal PK, Ebina W, Ben-Zvi A, Nombela-Arrieta C, Silberstein LE, et al. Fgd5 identifies hematopoietic stem cells in the murine bone marrow. J Exp Med. 2014;211:1315-31.

34. Sawai CM, Babovic S, Upadhaya S, Knapp DJHF, Lavin Y, Lau CM, et al. Hematopoietic stem cells are the major source of multilineage hematopoiesis in adult animals. Immunity. 2016;45:597-609.

35. Chapple RH, Tseng Y-J, Hu T, Kitano A, Takeichi M, Hoegenauer $\mathrm{KA}$, et al. Lineage tracing of murine adult hematopoietic stem cells reveals active contribution to steady-state hematopoiesis. Blood Adv. 2018;2:1220-8.

36. Tajima Y, Ito K, Umino A, Wilkinson AC, Nakauchi H, Yamazaki S. Continuous cell supply from Krt7-expressing hematopoietic stem cells during native hematopoiesis revealed by targeted in vivo gene transfer method. Sci Rep. 2017;7:1-10.

37. Genovese G, Kähler AK, Handsaker RE, Lindberg J, Rose SA, Bakhoum SF, et al. Clonal hematopoiesis and bloodcancer risk inferred from blood dna sequence. N Engl J Med. 2014;371:2477-87.

38. Jaiswal S, Fontanillas P, Flannick J, Manning A, Grauman PV, Mar BG, et al. Age-related clonal hematopoiesis associated with adverse outcomes. N Engl J Med. 2014;371:2488-98.

39. Sperling AS, Gibson CJ, Ebert BL. The genetics of myelodysplastic syndrome: from clonal haematopoiesis to secondary leukaemia. Nat Rev Cancer. 2017;17:5-19.

40. Van Der Weyden L, Giotopoulos G, Rust AG, Matheson LS, Van Delft FW, Kong J, et al. Modeling the evolution of ETV6RUNX1-induced B-cell precursor acute lymphoblastic leukemia in mice. Blood. 2011;118:1041-51.

41. Hong D, Gupta R, Ancliff P, Atzberger A, Brown J, Soneji S, et al. Initiating and cancer-propagating cells in TEL-AML1-associated childhood leukemia. Science (80-). 2008;319:336-9.

42. Papaemmanuil E, Rapado I, Li Y, Potter NE, Wedge DC, Tubio J, et al. RAG-mediated recombination is the predominant driver of oncogenic rearrangement in ETV6-RUNX1 acute lymphoblastic leukemia. Nat Genet. 2014;46:116-25.

43. Saida S, Watanabe KI, Sato-Otsubo A, Terui K, Yoshida K, Okuno Y, et al. Clonal selection in xenografted TAM recapitulates the evolutionary process of myeloid leukemia in Down syndrome. Blood. 2013;121:4377-87.

44. Roberts I, Alford K, Hall G, Juban G, Richmond H, Norton A, et al. GATA1-mutant clones are frequent and often unsuspected in babies with Down syndrome: identification of a population at risk of leukemia. Blood. 2013;122:3908-17.

45. Tarnawsky SP, Chan RJ, Yoder MC. Mice expressing Kras ${ }^{\mathrm{G} 12 \mathrm{D}_{\text {in }}}$ hematopoietic multipotent progenitor cells develop neonatal myeloid leukemia. J Clin Investig. 2017;127:3652-56.

46. Zhang J, Wang J, Liu Y, Sidik H, Young KH, Lodish HF, et al. Oncogenic Kras-induced leukemogeneis: hematopoietic stem cells as the initial target and lineage-specific progenitors as the potential targets for final leukemic transformation. Blood. 2009;113:1304-14.

47. Tang P, Gao C, Li A, Aster J, Sun L, Chai L. Differential roles of Kras and Pten in murine leukemogenesis. Leukemia. 2013;27:1210-14.

48. Tarnawsky SP, Yu W-M, Qu C-K, Chan RJ, Yoder MC. Hematopoietic-restricted Ptpn11E76K reveals indolent MPN progression in mice. Oncotarget. 2018;9:21831-43. 